Psychological Medicine, 2001, 31, 65-73. Printed in the United Kingdom

(C) 2001 Cambridge University Press

\title{
What contributes to depression in Parkinson's disease?
}

\author{
A. SCHRAG, M. JAHANSHAHI AND N. P. QUINN ${ }^{1}$ \\ From the Department of Clinical Neurology, Institute of Neurology, London
}

\begin{abstract}
Background. Depression is a common problem in patients with Parkinson's disease, but its mechanism is poorly understood. It is thought that neurochemical changes contribute to its occurrence, but it is unclear why some patients develop depression and others do not. Using a community-based sample of patients with Parkinson's disease, we investigated the contributions of impairment, disability and handicap to depression in Parkinson's disease.
\end{abstract}

Methods. Ninety-seven patients seen in a population-based study on the prevalence of Parkinson's disease completed the Beck Depression Inventory (BDI). Clinical and historical information on symptoms and complications of Parkinson's disease were obtained from the patients by a neurologist. In addition, clinician and patient ratings of disability on the Schwab and England scale were obtained and a quality of life questionnaire was completed.

Results. Moderate to severe depression (BDI $\geqslant 18$ ) was reported by $19 \cdot 6 \%$ of the patients. Higher depression scores were associated with advancing disease severity, recent self-reported deterioration, higher akinesia scores, a mini-mental score of $<25$ and occurrence of falls. Disability as rated by the neurologist accounted for $34 \%$ of the variance of depression scores. Self-reported impairment of cognitive function and the feeling of stigmatization accounted for $>50 \%$ of the variance of depression scores.

Conclusions. Depression in patients with Parkinson's disease is associated with advancing disease severity, recent disease deterioration and occurrence of falls. Regression analysis suggests that depression in Parkinson's disease is more strongly influenced by the patients' perceptions of handicap than by actual disability. The treatment of depression should therefore be targeted independently of treatment of the motor symptoms of Parkinson's disease, and consider the patients' own perception of their disease.

\section{INTRODUCTION}

Depression occurs more frequently in patients with Parkinson's disease than in the age-matched population (Warburton, 1967; Robins, 1976; Cantello et al. 1986; Gotham et al. 1986; Ehmann et al. 1990). It is presumed that in many cases depression is related to the neurochemical changes in Parkinson's disease (Mayeux et al. 1984, 1986; Ring et al. 1994; Charlton, 1997), but its mechanism is poorly understood, and it is unclear why some patients develop depression

${ }^{1}$ Address for correspondence: Professor Niall P. Quinn, Department of Clinical Neurology, Institute of Neurology, Queen Square, London WC1N 3BG. while others do not. There is no clear relationship between depression and severity or duration of Parkinson's disease or with the occurrence of specific parkinsonian symptoms (Brown \& Jahanshahi, 1995). In addition, except for depression as an off-period symptom, depression does not improve consistently with medical or surgical antiparkinsonian treatment (Lieberman, 1998; Quinn, 1998; Fetoni et al. 1999). Therefore, it has been suggested that personal and social factors (MacCarthy \& Brown, 1989), and reactions of the patient to the disease, play a role in determining depression in Parkinson's disease. However, disability and personal and social factors only account for $<50 \%$ of the 
variance of depression (MacCarthy \& Brown, 1989).

The World Health Organization (1980) has suggested a useful model to assess the impact of chronic disease on patients' lives. The objective impairment caused by the disease, such as presence and severity of symptoms, may lead to disability, the functional deficit resulting from impairment. Impairment and disability can both result in handicap, which depends on the patient's social and personal circumstances and his/her social roles and physical and emotional make-up. Although handicap usually results from the disability associated with impairment, it can result directly from impairment even without any accompanying disability (e.g. symptoms causing social embarrassment). Furthermore, patients with the same objective impairment (symptom severity) may experience very different degrees of disability and handicap. Mediating factors like personality traits or social circumstances can prevent or increase handicap in patients with the same level of disability or impairment. Thus, although these concepts overlap, they measure different aspects of the impact of illness and may therefore contribute differentially to depression.

We investigated some of the factors that may contribute to depression in a population-based sample of patients with Parkinson's disease. In the framework of the WHO model, we evaluated the relation of depression with various motor and non-motor symptoms of Parkinson's disease, with the associated disability, as measured by a clinician on the Schwab and England scale, and with self-reported handicap as indexed by a quality of life (QoL) measure.

\section{METHOD}

\section{Case ascertainment}

This study was part of a larger population-based study on the prevalence of parkinsonism with the primary aim of differentiating different types of parkinsonism. The methods of study design and case ascertainment have been reported previously (Schrag et al. 1999). In brief, the records of 15 general practices in the area of London were screened for patients with a suspicion of Parkinson's disease or parkinsonism, for patients with tremor with onset after the age of 50 , and for patients who had ever received antiparkinsonian drugs. As the health care system in the UK is organized locally the population of a general practice is representative of the surrounding area. In addition, the general practitioner acts as a 'gatekeeper' of the National Health Service, so that patients are only referred to specialists by their general practitioners. All participating patients had a semi-structured general and neurological interview and examination, and clinical details were collected during the diagnostic visit. Details of the patients' medical history were extracted from their general practitioners' records. Diagnosis of probable Parkinson's disease was made according to strict clinical diagnostic criteria (Gibb \& Lees, 1989). Patients with atypical parkinsonism, vascular parkinsonism, drug-induced parkinsonism, and those with parkinsonism following dementia were excluded. Overall, 242 patients fulfilled screening criteria and participation rate was high $(84 \%)$. Among all patients seen, 124 were diagnosed with idiopathic Parkinson's disease and were included in this study.

\section{Instruments}

The clinical assessment included the Hoehn and Yahr Disease Severity Scale (Hoehn \& Yahr, 1967), the Unified Parkinson's Disease Rating Scale (UPDRS; Fahn et al. 1987) the Schwab and England Disability Scale (Schwab \& England, 1969), and the Mini-Mental State Examination (MMSE; Folstein et al. 1975). In addition, all patients were asked to complete a questionnaire booklet and return it in a stamped, addressed envelope. This booklet comprised the Beck Depression Inventory, a widely used 21item depression inventory (BDI; Beck et al. 1961), and the PDQ-39, a disease-specific quality of life (QoL) instrument (Peto et al. 1995). The PDQ-39 comprises 39 questions each with five different answer options, from which eight subscores (mobility, activities of daily living, emotional well-being, stigma, social support, cognition, communication and bodily discomfort) can be derived, which can be totalled to give one summary index. The maximum score of 100 indicates the worst level of problem. On the BDI, higher scores (range 0-63) indicate more severe depression, and the cut-off score for moderate to severe depression used was 18 $(\geqslant 18$ indicates moderate to severe depression; 
Jahanshahi \& Marsden, 1988). Patients were also asked to rate their current general level of health compared with the past 12 months as better, much the same, or worse than on the day of completion.

\section{Statistical analysis}

Clinical and historical information on features and complications of PD were analysed with regard to their influence on depression scores. In addition, the association between depression scores and the results of the QoL questionnaire was investigated. Akinesia scores were calculated as the sum of items 19 and 23 to 26 of the UPDRS for both sides, and the tremor score from items 20 and 21 for both sides. The tremordominant subtype of PD was defined as patients with a ratio of tremor to bradykinesia score (subscores from the UPDRS motor scale and history) $\geqslant 0 \cdot 5$, and the akinetic-rigid subtype as patients with a ratio of $<0 \cdot 5$. The subdimensions and summary scores of the QoL instrument were calculated according to the respective scoring algorithms. The data were inspected for normality and equality of variance. Mean values were then compared with one-way analysis of variance, and Pearson rank correlations were calculated for examining associations of variables. Categorical data were analysed using the chi-square and Fisher's exact tests, where appropriate. Significance was accepted at the $1 \% \quad$ level $(P<0.01)$. Stepwise multiple regression analysis was used to determine the factors contributing independently to depression scores at a significance level of $P<0.05$.

\section{RESULTS}

Of the 124 patients $97(78 \%)$ returned the questionnaires. Their mean age was 73 (s.D. $11 \cdot 3$ ) years with a mean disease duration of 5.8 (S.D. 4.9) years, and a mean onset age of 67.6 (S.D. 12.5) years. There were 50 men and 47 women who responded, mean Hoehn and Yahr stage was $2 \cdot 4$ (S.D. 0.9), mean MMSE was $27 \cdot 5$ (out of 30; S.D. 3.6), and mean PDQ-39 score was $30 \cdot 0$ (S.D. 19.3). Seventeen patients (18\%) had MMS scores of $<25$. There was no statistical difference between responders and non-responders with respect to age, disease duration, sex, Hoehn and Yahr stage, Schwab and England score or MMSE score.

\section{Extent of depression}

The mean BDI score in this sample was 12 (S.D. 10.5; ranging from 0 to 54). Among the 97 responders $35(36 \cdot 1 \%)$ had mild (BDI score 9-17), $10(10 \cdot 3 \%)$ moderate (BDI score 18-23) and $9(9 \cdot 3 \%)$ severe depression (BDI score $>24$ ). Thus moderate to severe depression was reported by $19 \cdot 6 \%$ of the sample.

\section{The WHO model of impairment, disability and handicap}

To test the validity of this model to explain depression scores in patients with Parkinson's disease, we performed a factor analysis with varimax rotation using the different scales in this study. This resulted in two independent components which each accounted for $34 \%$ of the total variance. Factor one had loadings between 0.70 and 0.88 for all measures of impairment (Hoehn and Yahr stage, akinesia score, tremor score, MMSE score) as well as clinician- and patient-rated disability. Factor two had loadings between 0.51 and 0.82 for all measures of handicap (all subdimension of the PDQ-39 apart from the activities of daily living subdimension, which had a loading for factor one of 0.56 ). It therefore seems appropriate to interpret factor one as reflecting impairment and disability and factor two as reflecting handicap.

\section{Relation of depression to patient and disease characteristics}

Using Pearson correlations, there was a moderate, but significant, correlation of increasing depression scores with higher disease severity as measured by the Hoehn and Yahr scale $(r=$ $0 \cdot 36, P<0.001)$ and higher akinesia scores $(r=$ $0.37, P<0.01)$, and a trend for a correlation with higher tremor scores $(r=0.25, P<0.05)$, but not with age $(r=-0.02, P=0.9)$, age at onset $(r=-0.07, P=0.6)$ or disease duration $(r$ $=-0.02, P=0.8)$, even after controlling for disease severity. Using one-way analysis of variance, depression scores were significantly higher in patients who reported recent deterioration of their health $(P<0.001)$ and those with falls $(P<0.01)$ than for those without these features (see Table 1). There was also a trend for patients with the akinetic-rigid subtype of Parkinson's disease $(P<0.05)$, with postural instability $(P<0.05)$, a MMSE score 
Table 1. Factors associated with significantly increased depression scores

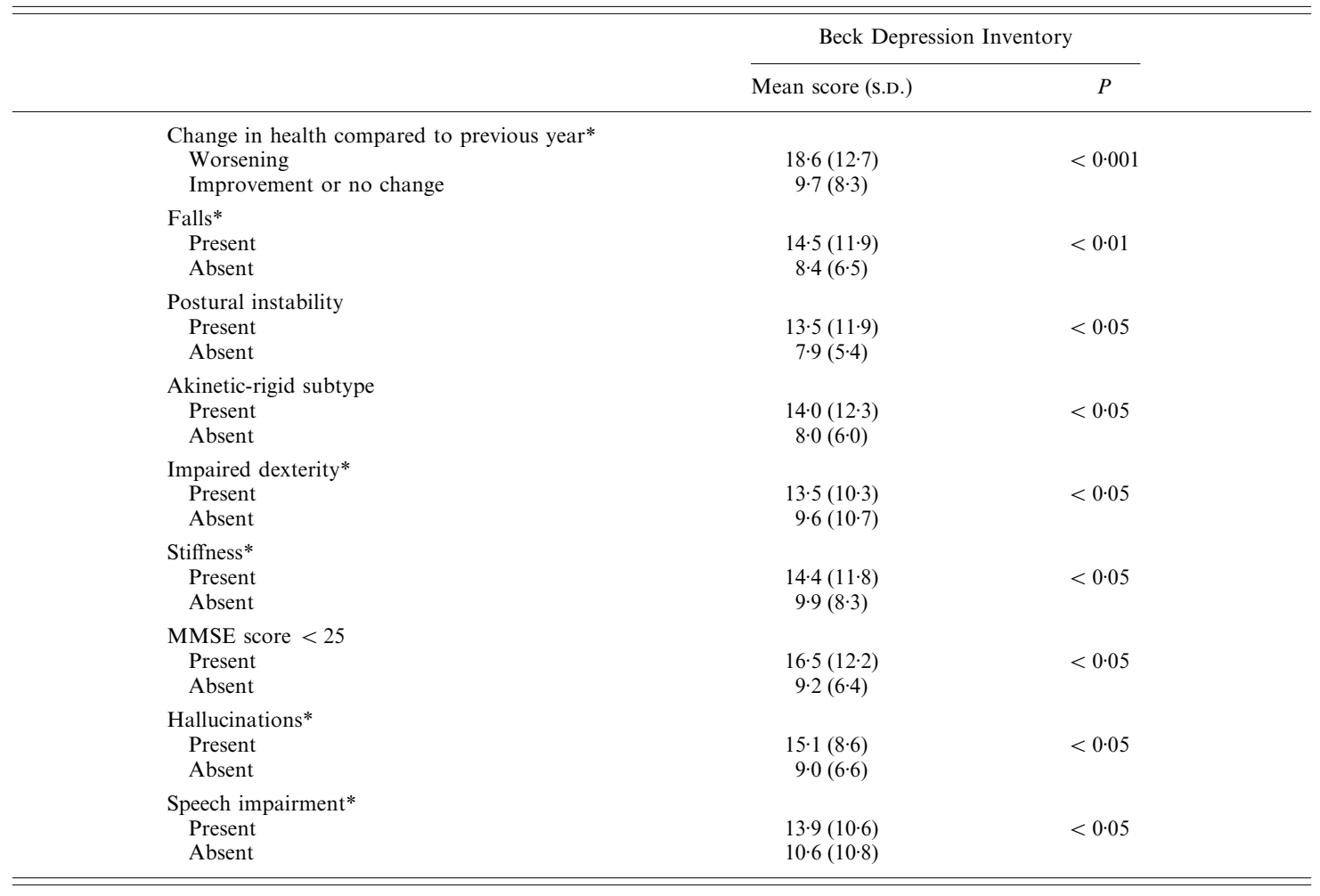

* Self-reported.

MMSE, Mini-Mental State Examination.

of $<25(P<0.05)$, self-reported hallucinations $(P<0.05)$, stiffness $(P<0.05)$ and impairment of dexterity $(P<0.05)$ or speech $(P<0.05)$, to have higher depression scores. In contrast, gender $(P=0 \cdot 1)$, onset before or after age $55(P$ $=0.9)$, current age above or below $60(P=0 \cdot 8)$, or the presence or absence of tremor $(P=0 \cdot 4)$ or rigidity $(P=0 \cdot 6)$, and self-report of dyskinesias $(P=0 \cdot 5)$, on-off fluctuations $(P=0 \cdot 3)$ or of response to levodopa $(P=0 \cdot 4)$ did not have a significant effect on depression scores.

A proportion of patients with moderate to severe depression were in the early stages of illness. However, overall more than $90 \%$ of patients with depression scores of 18 or more had Hoehn and Yahr stages of 3, 4 or 5. Also, patients with BDI scores of $\geqslant 18$ had significantly greater postural instability, as measured on the UPDRS motor scale $(P<0 \cdot 001)$, more frequently reported recent deterioration of their health $(P<0.005)$, and tended to have lower MMSE scores $(P<0.01$; Table 2$)$. Overall,
$47 \%$ of patients with MMSE scores of $\leqslant 24$ compared with $14 \%$ of those with MMSE scores of $\geqslant 25$ had BDI scores of $\geqslant 18$. No significant difference was found between depressed and non-depressed patients with regard to age $(P=0 \cdot 7)$, sex $(P=1 \cdot 0)$ or age at onset $(P$ $=0 \cdot 2)$, tremor $(P=0.6)$, rigidity $(P=0.9)$ and akinesia scores $(P=0 \cdot 1)$, presence/absence of fluctuations $(P=0 \cdot 3)$, dyskinesias $(P=0 \cdot 3)$ or hallucinations $(P=0 \cdot 1)$, socio-economic status $(P=0 \cdot 2)$ or living conditions $(P=0 \cdot 3)$.

\section{Relation of depression to disability}

Patients with BDI scores of $\geqslant 18$ had significantly greater disability scores than those with scores of $<18$ as rated by the clinician $(P=$ $0.002)$ or the patient $(P=0.0001$; Table 2$)$. Depression scores increased with decreasing Schwab and England scores, indicating greater depression with increased disability, whether assessed by the clinician $(r=-0.57, P<0 \cdot 0001)$, or by the patient $(r=-0 \cdot 60, P<0 \cdot 0001)$. There 
Table 2. Comparison of the depressed $(B D I>17)$ and non-depressed patients on measures of impairment, disability and handicap (standard deviations are in parentheses)

\begin{tabular}{|c|c|c|c|}
\hline Variable & $\begin{array}{l}\text { Depressed } \\
\text { Mean (s.D.) }\end{array}$ & $\begin{array}{l}\text { Non-depressed } \\
\text { Mean (s.D.) }\end{array}$ & $P<$ \\
\hline \multicolumn{4}{|l|}{ Impairment } \\
\hline Postural instability* & $1.9(0.8)$ & $1 \cdot 1(0 \cdot 7)$ & 0.001 \\
\hline MMSE & $25 \cdot 7(5 \cdot 6)$ & $27 \cdot 9(3 \cdot 5)$ & $0 \cdot 01$ \\
\hline Self-reported recent deterioration & $61.5 \%$ & $20 \%$ & 0.005 \\
\hline \multicolumn{4}{|l|}{ Disability: Schwab and England score } \\
\hline Clinician-rated & $71 \cdot 4(22 \cdot 4)$ & $83 \cdot 6(14 \cdot 0)$ & 0.002 \\
\hline Patient-rated & $48 \cdot 0(29 \cdot 0)$ & $79 \cdot 0(16 \cdot 4)$ & 0.0001 \\
\hline \multicolumn{4}{|l|}{ Handicap: PDQ-39 } \\
\hline Cognition & $52 \cdot 9(23 \cdot 5)$ & $25 \cdot 3(18 \cdot 3)$ & $0 \cdot 00001$ \\
\hline Bodily pain & $50 \cdot 1(26 \cdot 9)$ & $32 \cdot 6(19 \cdot 0)$ & 0.0005 \\
\hline Communication & $35 \cdot 4(28 \cdot 0)$ & $15 \cdot 3(17 \cdot 1)$ & $0 \cdot 0001$ \\
\hline Emotional well-being & $46 \cdot 0(25 \cdot 8)$ & $23 \cdot 2(16 \cdot 6)$ & 0.0005 \\
\hline Social support & $29 \cdot 4(26 \cdot 1)$ & $10 \cdot 2(16 \cdot 3)$ & 0.001 \\
\hline Activities of daily living & $51 \cdot 1(29 \cdot 4)$ & $33 \cdot 1(26 \cdot 6)$ & 0.003 \\
\hline Stigma & $32 \cdot 3(29 \cdot 7)$ & $19 \cdot 0(25 \cdot 4)$ & 0.004 \\
\hline Mobility & $63 \cdot 5(31 \cdot 1)$ & $45 \cdot 5(32 \cdot 0)$ & 0.004 \\
\hline
\end{tabular}

* Measured on the Unified Parkinson's Disease Rating Scale (Fahn et al. 1987).

MMSE, Mini-Mental State Examination (Folstein et al. 1975); PDQ-39, Parkinson's disease specific quality of life questionnaire (Peto et al. 1995).

was a strong correlation between the clinician's and the patient's rating of disability on the Schwab and England scale $(r=0 \cdot 89$, $P<0.0001)$. However, patients with BDI scores of $\geqslant 18$ rated their disability greater than the clinician, whereas no difference between clinician and patient-rated disability was seen in patients with BDI scores below 18 (two-factor mixed ANOVA $F(1,95)=15 \cdot 3, P<0 \cdot 001)$.

Relation of depression to self-rated health status Depression scores were most closely related to subjectively perceived impairment of cognition, as measured on the PDQ-39, $\quad(r=0 \cdot 67$, $P<0 \cdot 0001)$. They were also significantly correlated with impairment of all other aspects of QoL, although less strongly with impairment of social support, as measured on the PDQ-39 $(r=$ $0 \cdot 32, P<0 \cdot 01)$. The patients with a BDI score of $\geqslant 18$ had significantly worse scores on all areas of QoL (see Table 2). Even when controlling for disability as measured by the clinician-rated Schwab and England score, BDI scores correlated with all areas of QoL, albeit less significantly.

\section{Predictors of depression in Parkinson's disease: multiple regression analysis}

To determine the factors that contributed most to depression, we performed a series of stepwise multiple regression analyses entering all factors that had shown a significant and sizeable correlation with depression scores or resulted in significantly different depression scores. In the context of the WHO model, we performed separate analyses for measures of impairment, disability and handicap. First, parameters measuring impairment (Hoehn and Yahr stage, akinesia score, postural instability, MMSE score and recent deterioration) were entered. Secondly, in a separate analysis, disability scores, as measured by the Schwab and England scale by the clinician and by the patient, were entered. Thirdly, a stepwise multiple regression was performed with QoL scores as the independent variables to detemine which specific aspects of handicap related most closely to depression. Finally, an overall analysis was carried out, using measures of impairment, disability and handicap in a single regression analysis, to determine the best overall predictors of depression.

\section{Disease characteristics - degree of impairment}

Disease severity, as measured on the Hoehn and Yahr scale, accounted for $19 \%$ of the variance in BDI scores, and the self-report of recent deterioration for an additional $11 \%$. Together they explained $28 \%$ of the variance of depression 
scores (adjusted $R^{2}=0 \cdot 28, P<0 \cdot 001$ ). No other disease-related factor or patient characteristic additionally contributed to the prediction of depression scores.

\section{Disability}

Disability, as measured by the clinician-rated Schwab and England scores, explained $34 \%$ of the variance of depression $(P<0 \cdot 0001)$. Patientrated Schwab and England scores alone explained $51 \%$ of the variance of depression $(P<0.0001)$.

\section{Quality of life}

Stepwise multiple regression with QoL subscores revealed that impairment in the areas of cognition, emotional well-being, stigma, and activities of daily living (as measured on the PDQ39) predicted depression scores in $66 \%$ of patients $(P<0.0001)$. When the domain that overlaps with depression (emotional well-being) was excluded from the analysis, subjective impairment of cognition (adjusted $R^{2}=-0 \cdot 47$, $P<0.0001)$, and the feeling of stigmatization, ( $R^{2}$ change $0.08, P<0.0001$ ), still predicted $53 \%$ of the variance of depression scores.

Overall: impairment, disability and $Q o L$

When measures of impairment, disability and QoL (excluding emotional well-being) were all entered in a stepwise regression analysis, impairment of cognition (adjusted $R^{2}=0 \cdot 47$, $P<0.0001)$ and stigmatization $\left(R^{2}\right.$ change $=$ $0.08, P<0.003)$, as measured on the PDQ-39, the clinician-rated disability $\left(R^{2}\right.$ change $=0.05$, $P=0.006)$, and the patient's report of recent deterioration $\left(R^{2}\right.$ change $\left.=0.04, \quad P=0.014\right)$ accounted for $60 \%$ of the variance of depression scores.

\section{DISCUSSION}

\section{Prevalence of depression in Parkinson's disease}

Moderate to severe depression with BDI scores of $\geqslant 18$ was seen in $19 \cdot 6 \%$ of patients with Parkinson's disease in a population-based sample. Studies in clinic-based samples or using different instruments have reported a wide range of depression rates (Celesia \& Wanamaker, 1972; Mayeux et al. 1981; Cummings, 1992), but in the only comparable, population-based study in Sweden a similar rate of $24 \%$ was found when using the same instrument and cut-off score, although the authors diagnosed major depression in only $7.7 \%$ of patients in that sample (Tandberg et al. 1996). We considered the BDI a suitable instrument to measure the severity of depression in patients with Parkinson's disease in this study and to examine the influence of factors contributing to depression for several reasons. First, it is widely used and has been repeatedly shown to be valid in patients with Parkinson's disease. Secondly, it enables quantitative assessment of the severity of depression. Thirdly, its validity and internal consistency have been shown to be comparable in patients with Parkinson's disease and controls (Levin et al. 1988).

\section{Factors contributing to depression in Parkinson's disease}

We used the WHO model of impairment, disability and handicap to investigate factors contributing to depression in Parkinson's disease. Although there is obvious overlap between these concepts, impairment reflects objectively measured clinical symptoms and signs, whereas disability measures the individual's functioning in his environment, and handicap depends on the individual's perception of the impact of illness on his/her life influenced by a variety of personal, social and cultural factors. Thus, each of these concepts is useful in its own right to assess the effect of Parkinson's disease on an individual, thus differentially contributing to the understanding of depression in Parkinson's disease. In order to test the validity of this model, a factor analysis with the different scales used was performed and confirmed the existence of two separate dimensions, one relating to measures of impairment and disability, and the other to measures of handicap. Disability was not sufficiently different from measures of impairment to result in an independent factor. As there was a strong correlation between Hoehn and Yahr stage and the Schwab and England scale, which is mainly based on physical functioning, it is not surprising that they related to the same independent factor. However, the Schwab and England scale was the only measure of disability used, and the inclusion of other measures of disability may have resulted in a third independent factor.

Among measures of impairment, greater 
disease severity was overall associated with higher depression scores, but the prevalence of depression had a bimodal distribution, with a percentage of patients being depressed at initial stages of the disease (Hoehn and Yahr stage $1 \cdot 5)$, and a larger percentage at later stages (4 and 5). This finding has also been previously reported (Celesia \& Wanamaker, 1972; Starkstein et al. 1990), and may indicate reactive depression soon after diagnosis, improvement of depression in the process of adaptation to a slowly advancing disease and improvement in parkinsonian features after initial treatment, and subsequent recurrence of depression as the disease and its response to medication deteriorates.

In accordance with previous reports, subgroups of patients with the akinetic-rigid subtype of Parkinson's disease (Starkstein et al. 1998) and those with cognitive impairment (Starkstein et al. 1990) were more likely to be depressed than those without these characteristics. In addition, we found that postural instability and recent deterioration were also associated with higher depression scores, whereas age, type of onset, sex, age and the presence of fluctuations or dyskinesias did not influence patients' overall mood.

However, measures of impairment only predicted a small percentage of the variance of depression (28\%). Objective disease severity, as rated on the Hoehn and Yahr scale, predicted $19 \%$ of the variance of depression scores, which is similar to the findings of previous studies (Brown \& Jahanshahi, 1995). The consistent finding that only a small proportion of the variance of depression scores can be predicted by disease severity suggests that the development of mood disorder may be determined by a separate mechanism from that underlying the motor dysfunction in Parkinson's disease.

Although no other disease-related factors contributed to prediction of depression scores, the patients' own report of a recent deterioration in their condition accounted for a further $11 \%$ of the variance of depression scores. In this cross-sectional study, we did not assess disease progression over time objectively, and selfreported deterioration of the motor symptoms may be influenced by depressed patients' pessimistic view of their condition. Thus, the direction of effect cannot be clearly established. However, this finding is in agreement with previous studies which found that deterioration is an important factor in developing depression in Parkinson's disease (Brown et al. 1989; Starkstein et al. 1998), and with studies in other chronic diseases, where a progressive course is associated with higher depression scores (Moos \& Solomon, 1965; Mindham et al. 1981; McIvor et al. 1984; Starkstein et al. 1992).

Clinician-rated disability, on the other hand, correlated strongly with depression and was the strongest single predictor of depression in Parkinson's disease in this study. It explained $34 \%$ of the variance of the depression scores, which is more than twice that found in previous studies (Gotham et al. 1986; MacCarthy \& Brown, 1989; Ehmann et al. 1990). As may be expected, the patients' own rating of disability correlated even more strongly with their depression scores, but again the direction of effect is unclear and it is possible that presence of depression colours the patients' perceptions of disability negatively. This is supported by the finding that depressed patients had significantly greater self-rated than clinician-rated disability, whereas there was no such difference in nondepressed patients. However, this confounding factor is not applicable to the clinician-rated disability scores.

In this study, although disability was the single most influential predictor of depression, the degree of depression was best predicted by considering the patients' own judgement of their handicap, which explained more than $50 \%$ of its variance. Once again, it may be argued that selfreport of poor QoL may reflect the pessimistic outlook on the future by depressed patients, and thus depression may be influencing self-rated QoL scores rather than the reverse. However, the pattern and uneven distribution of correlations suggests that this is not the full explanation. Among the subjectively evaluated areas of health and QoL, impairment of mobility, as measured by mobility and activities of daily living scores on the PDQ-39, was least related to depression. More influential were the patients' reports of cognitive impairment and of stigmatization. Even if the direction of effect is the reverse and thus the results indicate that a depressed Parkinson's disease patient views his quality of life worse than a non-depressed Parkinson's disease patient, the greater con- 


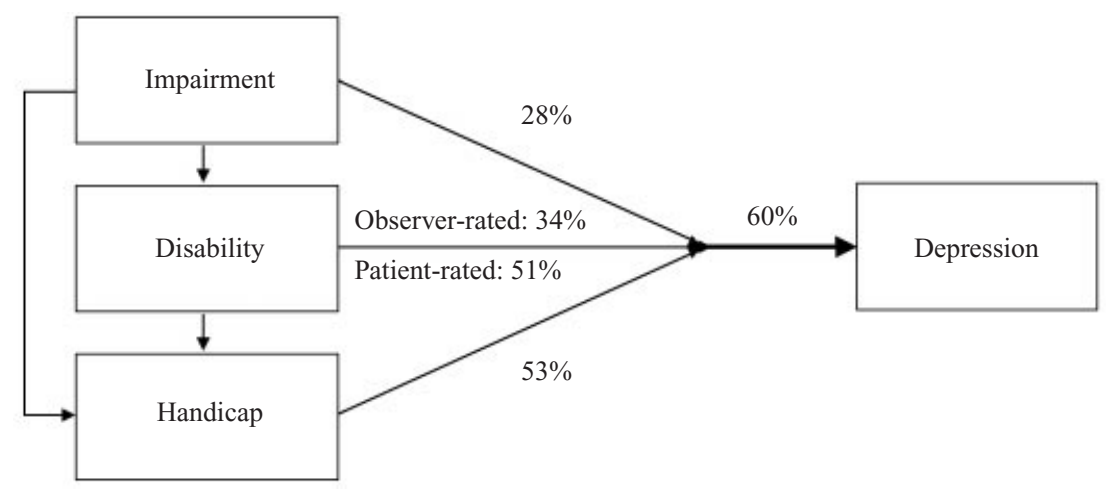

FIG. 1. The contribution of impairment, disability and handicap to depression in Parkinson's disease.

tribution of cognitive impairment and social stigmatization emphasizes the importance of these factors for the patients' well-being beyond the degree of physical impairment.

This study has several limitations. Depression was measured using the BDI, but we did not perform a structured psychiatric assessment. The prevalence rate therefore relates to the proportion of patients scoring highly on that scale rather than the presence of a depressive illness. Self-assessment may however be unreliable, particularly in demented patients. Furthermore, social functioning, social ties and other social, economic, personal and cultural issues were not assessed in this study. As these are likely to play an important role in the occurrence of depression, a higher proportion of the variance of depression scores might have been explained had these factors been measured.

Overall, in this study depression in Parkinson's disease was found to be the result of a multifactorial process, with objective impairment explaining only a comparatively small percentage of depression scores. Disability accounted for a greater proportion of the variability of depression scores, but the best predictor of depression was the patients' handicap, as measured by a health-related QoL instrument. Handicap, as indexed by a QoL measure, predicted more than $50 \%$ of the variance of depression scores (see Fig. 1). A strong association of depression with self-reported handicap, in the context of its less strong association with measures of disease severity, supports the notion that depression is at least partly a reaction to the impact of the disease on the individual's QoL. In contrast, from their population-based study of depression in patients with Parkinson's disease, Tandberg et al. (1997) concluded that depression is a primary consequence of brain dysfunction. However, in their study, the only parameter assessing personal and social factors was nursing-home placement, and the patients' perceptions were not considered. We therefore propose that, without disputing the known biochemical changes that may contribute to depression in Parkinson's disease, the patients' own personal circumstances and perception also influence its occurrence. The clinical differences between depressed and non-depressed patients (Table 1) emphasise the complexity of this model, and suggest that subgroups of patients, such as those with postural instability and cognitive decline, may be more predisposed to developing depression.

In conclusion, we found that patients with cognitive impairment or postural instability, and those with recent deterioration, were more likely to report themselves as being moderately to severely depressed than those without these disease features. However, depression in patients with Parkinson's disease appears to be multifactorial. In addition to the biochemical changes that undoubtedly play a role in the genesis of depression in Parkinson's disease, the patients' perception of handicap, i.e. the impact of the disease on their lives, appears more important than the severity and degree of symptomatic impairment. In practical terms, this underlines the importance of addressing the social and personal circumstances and the expectations and perceptions of a patient with Parkinson's disease who is depressed, and of treating his or her depression independently, rather than fo- 
cusing treatment exclusively on the motor symptoms of Parkinson's disease.

The financial support of SmithKline Beecham Pharmaceuticals (A. S.) and the Wellcome Trust (M. J.) is gratefully acknowledged.

\section{REFERENCES}

Beck, A. T., Ward, C., Mendelson, M., Mock, J. \& Erbaugh, J. (1961). An inventory for measuring depression. Archives of General Psychiatry 4, 561-567.

Brown, R. \& Jahanshahi, M. (1995). Depression in Parkinson's disease: a psychosocial viewpoint. Advances in Neurology 65, 61-84.

Brown, R. G., MacCarthy, B., Jahanshahi, M. \& Marsden, C. D (1989). Accuracy of self-reported disability in patients with parkinsonism. Archives of Neurology 46, 955-959.

Cantello, R., Gilli, M., Riccio, A. \& Bergamasco, B. (1986). Mood changes associated with 'end-of-dose deterioration' in Parkinson's disease: a controlled study. Journal of Neurology, Neurosurgery and Psychiatry 49, 1182-1190.

Celesia, G. G. \& Wanamaker, W. M. (1972). Psychiatric disturbances in Parkinson's disease. Diseases of the Nervous System 33, 577-583.

Charlton, C. G. (1997). Depletion of nigrostriatal and forebrain tyrosine hydroxylase by S-adenosylmethionine: a model that may explain the occurrence of depression in Parkinson's disease. Life Sciences 61, 495-502.

Cummings, J. L. (1992). Depression and Parkinson's disease: a review. American Journal of Psychiatry 149, 443-454.

Ehmann, T. S., Beninger, R. J., Gawel, M. J. \& Riopelle, R. J. (1990). Depressive symptoms in Parkinson's disease: a comparison with disabled and control subjects. Journal of Geriatric Psychiatry and Neurology 3, 3-9.

Fahn, S., Elton, R. L. \& Members of the UPDRS Development Committee (1987). Unified Parkinson's Disease Rating Scale. In Recent Developments in Parkinson's Disease, Vol. 2 (ed. S. Fahn, C. D. Marsden, D. B. Calne and M. Goldstein), pp. 153-163, MacMillan Healthcare Information: Florham Park, NJ.

Fetoni, V., Soliveri, P., Monza, D., Testa, D. \& Girotti, F. (1999). Affective symptoms in multiple system atrophy and Parkinson's disease: response to levodopa therapy. Journal of Neurology, Neurosurgery and Psychiatry 66, 541-544.

Folstein, M. F., Folstein, S. E. \& McHugh, P. R. (1975). 'Minimental state'. A practical method for grading the cognitive state of patients for the clinician. Journal of Psychiatric Research 12, 189-198.

Gibb, W. R. \& Lees, A. J. (1989). The significance of the Lewy body in the diagnosis of idiopathic Parkinson's disease. Neuropathology and Applied Neurobiology 15, 27-44.

Gotham, A. M., Brown, R. G. \& Marsden, C. D. (1986). Depression in Parkinson's disease: a quantitative and qualitative analysis. Journal of Neurology, Neurosurgery and Psychiatry 49, 381-389.

Hoehn, M. M. \& Yahr, M. D. (1967). Parkinsonism: onset, progression and mortality. Neurology 17, 427-442.

Jahanshahi, M. \& Marsden, C. D. (1988). Depression in torticollis: a controlled study. Psychological Medicine 18, 925-933.

Levin, B. E., Llabre, M. M. \& Weiner, W. J. (1988). Parkinson's disease and depression: psychometric properties of the Beck Depression Inventory. Journal of Neurology Neurosurgery and Psychiatry 51, 1401-1404.

Lieberman, A. (1998). Managing the neuropsychiatric symptoms of Parkinson's disease. Neurology 50 (suppl. 6), S33-S38.
MacCarthy, B. \& Brown, R. (1989). Psychosocial factors in Parkinson's disease. British Journal of Clinical Psychology 28, 41-52.

McIvor, G. P., Riklan, M. \& Reznikoff, M. (1984). Depression in multiple sclerosis as a function of length and severity of illness, age, remissions, and perceived social support. Journal of Clinical Psychology 40, 1028-1033.

Mayeux, R., Stern, Y., Rosen, J. \& Leventhal, J. (1981). Depression, intellectual impairment, and Parkinson disease. Neurology 32, 645-650.

Mayeux, R., Stern, Y., Cote, L. \& Williams, J. B. (1984). Altered serotonin metabolism in depressed patients with Parkinson's disease. Neurology 34, 642-646.

Mayeux, R., Stern, Y., Williams, J. B., Cote, L., Frantz, A. \& Dyrenfurth, I. (1986). Clinical and biochemical features of depression in Parkinson's disease. American Journal of Psychiatry 143, 756-759.

Mindham, R. H., Bagshaw, A., James, S. A. \& Swannell, A. J. (1981). Factors associated with the appearance of psychiatric symptoms in rheumatoid arthritis. Journal of Psychosomatic Research 25, 429-435.

Moos, R. H. \& Solomon, G. F. (1965). Personality correlates of the degree of functional incapacity of patients with physical disease. Journal of Chronic Disease 18, 1019-1038.

Peto, V., Jenkinson, C., Fitzpatrick, R. \& Greenhall, R. (1995). The development and validation of a short measure of functioning and well being for individuals with Parkinson's disease. Quality of Life Research 4, 241-248.

Quinn, N. P. (1998). Classification of fluctuations in patients with Parkinson's disease. Neurology 51 (suppl. 2), S25-S29.

Ring, H. A., Bench, C. J., Trimble, M. R., Brooks, D. J., Frackowiak, R. S. \& Dolan, R. J. (1994). Depression in Parkinson's disease. A positron emission study. British Journal of Psychiatry 165, 333-339.

Robins, A. H. (1976). Depression in patients with Parkinsonism. British Journal of Psychiatry 128, 141-145.

Schrag, A., Ben-Shlomo, Y. \& Quinn, N. P. ( 1999). The population prevalence of progressive supranuclear palsy and multiple system atrophy. Lancet 354, 171-175.

Schwab, R. S. \& England, A. C. (1969). Projection technique for evaluating surgery in Parkinson's disease. In Third Symposium on in Parkinson's Disease (ed. F. J. Gillingham and I. M. L. Donaldson), pp. 152-157. Livingstone: Edinburgh.

Starkstein, S. E., Preziosi, T. J., Bolduc, P. L. \& Robinson, R. G. (1990). Depression in Parkinson's disease. Journal of Nervous and Mental Disease 178, 27-31.

Starkstein, S. E., Mayberg, H. S., Leiguarda, R., Preziosi, T. J. \& Robinson, R. G. (1992). A prospective longitudinal study of depression, cognitive decline, and physical impairments in patients with Parkinson's disease. Journal of Neurology, Neurosurgery and Psychiatry 55, 377-382.

Starkstein, S. E., Petracca, G., Chemerinski, E., Teson, A., Sabe, L., Merello, M. \& Leiguarda, R. (1998). Depression in classic versus akinetic-rigid Parkinson's disease. Movement Disorders 13, 29-33.

Tandberg, E., Larsen, J. P., Aarsland, D. \& Cummings, J. L. (1996). The occurrence of depression in Parkinson's disease. A communitybased study. Archives of Neurology 53, 175-179.

Tandberg, E., Larsen, J. P., Aarsland, D., Laake, K. \& Cummings, J. L. (1997). Risk factors for depression in Parkinson disease. Archives of Neurology 54, 625-630.

Warburton, J. W. (1967). Depressive symptoms in Parkinson patients referred for thalamotomy. Journal of Neurology, Neurosurgery and Psychiatry 30, 368-370.

World Health Organization (1980). International Classification of Impairments, Disabilities and Handicaps. A Manual of Classification Relating to the Consequences of Disease. World Health Organisation: Geneva. 